\title{
Evidence for the involvement of ZNF804A in cognitive processes of relevance to reading and spelling
}

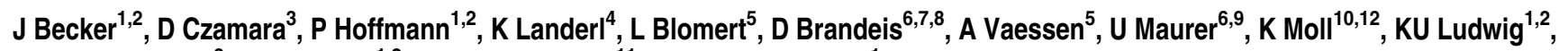 \\ B Müller-Myhsok ${ }^{3}$, MM Nöthen ${ }^{1,2}$, G Schulte-Körne $^{11}$ and J Schumacher ${ }^{1}$
}

Previous studies have shown that individuals with schizophrenia and dyslexia display common neurocognitive abnormalities. The aim of the present study was to determine whether known schizophrenia-risk genes contribute to dyslexia risk or to diseaserelevant cognitive functions. For this purpose, we genotyped the schizophrenia-associated risk variants within zinc-finger protein $804 A$ (ZNF804A), transcription-factor 4 and neurogranin in a large dyslexia case-control sample. We tested all variants for association with dyslexia (927 cases, 1096 controls), and with eight language-relevant cognitive processes (1552 individuals). We observed six significant associations between language-relevant traits and the ZNF804A-variant rs1344706. Interestingly, the ZNF804A schizophrenia risk variant was associated with a better cognitive performance in our data set. This finding might be consistent with a previously reported ZNF804A association in schizophrenia, in which patients carrying the schizophrenia-risk allele at rs 1344706 showed a better performance in two memory tests. In conclusion, the present study provides evidence that ZNF804A might have a role in cognitive traits of relevance to reading and spelling, and underlines the phenotypic complexity that might be associated with ZNF804A.

Translational Psychiatry (2012) 2, e136; doi:10.1038/tp.2012.62; published online 10 July 2012

\section{Introduction}

Several genome-wide association studies have been performed for schizophrenia to date, and zinc-finger protein $804 \mathrm{~A}$ (ZNF804A), transcription-factor 4 (TCF4) and neurogranin (NRGM) were among the first genes to achieve genome-wide significance. ${ }^{1,2}$ These findings have since been replicated in independent samples, and these genes are therefore likely to represent true risk factors for schizophrenia. ${ }^{3}$

Given that individuals with dyslexia and schizophrenia have been reported to display certain common neurocognitive abnormalities, ${ }^{4,5}$ we were interested in determining whether these genes contribute to dyslexia risk or to disease-relevant cognitive functions. For this purpose, we genotyped the identified schizophrenia risk variants in ZNF804A (rs1344706), TCF4 (rs9960767) and NRGN (rs12807809) in five dyslexia case-control samples of Central European descent. Subsequently, we performed association analyses using case-control status (927 cases and 1096 controls) and eight dyslexia-relevant psychometric tests (1552 individuals). The psychometric tests encompass the dyslexia core symptoms of impaired reading and spelling (word reading, word spelling), phonological awareness (phoneme deletion), phonological coding (non-word reading), auditory short-term memory (digit span) and rapid naming (letters, digits, pictures).

\begin{abstract}
Materials and methods
We investigated five dyslexia case-control samples from Salzburg (Austria), Zurich (Switzerland), Marburg (Germany), Munich (Germany) and Maastricht (The Netherlands). The whole sample set was comprised of 927 dyslexia cases and 1096 controls. Besides case-control status, data on eight language-relevant cognitive processes were available for all individuals, with the exception of the Marburg-controls $(N=1552$, Supplementary Table 1$)$. These eight cognitive processes include word reading, word spelling, phonological awareness (phoneme deletion), phonological coding (nonword reading), auditory short-term memory (digit span) and rapid naming (letters, digits, pictures). Details of the phenotypic measures are provided in Supplementary Table 2.

Genotyping of the variants of interest was carried out on the Sequenom MassArray system (Sequenom, San Diego, CA, USA). Primer sequences and standard assay conditions are available upon request.
\end{abstract}

\footnotetext{
${ }^{1}$ Institute of Human Genetics, University of Bonn, Bonn, Germany; ${ }^{2}$ Department of Genomics, Life and Brain Center, University of Bonn, Bonn, Germany; ${ }^{3}$ Max Planck Institute of Psychiatry, Munich, Germany; ${ }^{4}$ Department of Psychology, University of Graz, Graz, Austria; ${ }^{5}$ Department of Cognitive Neuroscience, Faculty of Psychology and Neuroscience, Maastricht Brain Imaging Institute (M-BIC), Maastricht University, Maastricht, The Netherlands; ${ }^{6}$ Department of Child and Adolescent Psychiatry, University of Zurich, Zurich, Switzerland; ${ }^{7}$ Zurich Center for Integrative Human Physiology (ZIHP), Zurich, Switzerland; ${ }^{8}$ Department of Child and Adolescent Psychiatry and Psychotherapy, Central Institute of Mental Health, Medical Faculty Mannheim/Heidelberg University, Mannheim, Germany; ${ }^{9}$ Department of Psychology, University of Zurich, Zurich, Switzerland; ${ }^{10}$ Department of Psychology, University of Salzburg, Salzburg, Austria and ${ }^{11}$ Department of Child and Adolescent Psychiatry and Psychotherapy, University Hospital Munich, Munich, Germany

Correspondence: Dr Johannes Schumacher, Institute of Human Genetics, University of Bonn, Sigmund-Freud-Strasse 25, D-53105 Bonn, Germany.

E-mail: johannes.schumacher@uni-bonn.de

${ }^{12}$ Current address: Department of Psychology, University of York, York, UK.

Keywords: cognitive processes; dyslexia; genetic association; reading and spelling; schizophrenia; ZNF804A

Received 27 Feburary 2012; revised 15 May 2012; accepted 31 May 2012
} 
Association analyses were performed using PLINK. ${ }^{6}$ Logistic regression was used for case-control analyses, and linear regression was used for quantitative trait analyses. To avoid false-positive results due to possible population stratification in our sample set, we performed an inverse variance weighted fixed effects meta-analysis using the Rlibrary rmeta (http://www.r-project.org/).

\section{Results}

In 927 dyslexia cases and 1096 controls, no dyslexia association was found for TCF4 or NRGN (Supplementary Tables 3 and 4). Although the association between rs 1344706 in ZNF804A and dyslexia was not statistically significant, we observed a similar genetic effect size (odds ratio $=1.12$, Table 1) as that reported previously in patients with schizophrenia (odds ratio $=1.09$ ). ${ }^{1}$ Notably, the $Z N F 804 A$ schizophrenia-protective allele was more common in dyslexia patients.

In a second step, we combined all cases and controls (1552 individuals) and tested whether the schizophrenia-risk genes were involved in language-relevant cognitive processes. Negative results were obtained for the variants in TCF4 and NRGN (Supplementary Tables 3 and 4). In contrast, rs1344706 in ZNF804A showed association with six phenotypes (Table 1). Here, the schizophrenia-protective allele and the schizophrenia-risk allele were consistently associated with a poorer and a better performance in all cognitive tests, respectively. After correction for multiple testing, the associations with word reading, non-word reading and rapid naming of digits remained significant. Forest plots of rs 1344706 for the case-control analysis and eight quantitative trait analyses are provided in Supplementary Figure 1. Furthermore, we performed a heterogeneity analysis and observed no differences between the rs1344706 effect sizes for the five subsamples: Salzburg, Zurich, Marburg, Munich and Maastricht $(P>0.05$, Supplementary Table 5). The estimated effect sizes for the association between rs 1344706 and the cognitive phenotypes are shown in Supplementary Table 6. Finally, we tested whether rs1344706 was associated with the intelligence quotient level, and observed no association (Supplementary Table 7).

\section{Discussion}

The present findings provide evidence that ZNF804A might be involved in cognitive functions of relevance to reading and spelling. This hypothesis might be supported by the 2q31.22q32.3-deletion syndrome phenotype, which includes severe speech impairment. ${ }^{7}$ ZNF804A is one of the few genes present within the deleted region, and previous authors have speculated that the deletion in ZNF804A may influence the speech phenotype in 2q31.2-2q32.3-syndrome patients. ${ }^{7}$ Furthermore, our data provide evidence that the allele at ZNF804A-rs 1344706 that influences the risk for schizophrenia might be associated with a better performance in language-relevant processes. In contrast, the schizophrenia-protective allele at rs 1344706 appeared to be associated with a poorer performance in cognitive processes of relevance to reading and spelling. A similar effect was observed in a

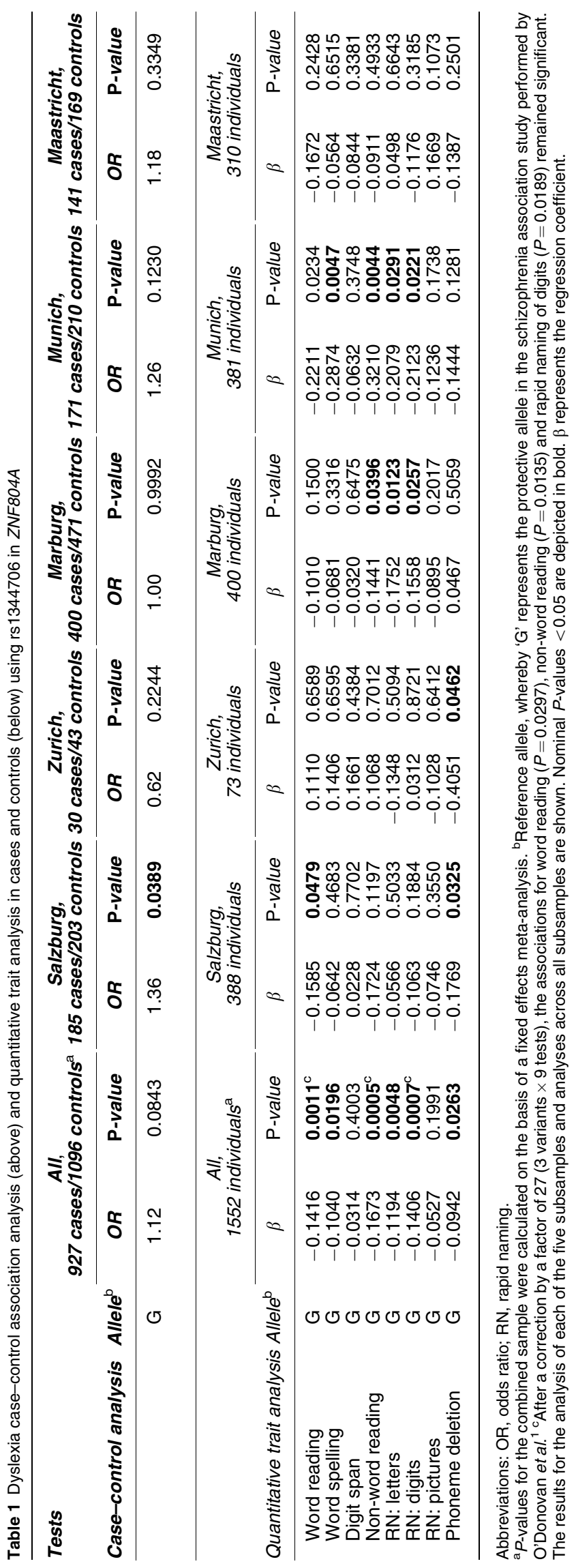


previous study, which analyzed cognitive profile in two schizophrenia samples. ${ }^{8}$ In that study, patients carrying the ZNF804A schizophrenia-risk allele also showed a significantly better performance in two memory tests compared with patients carrying the protective allele. However, it is difficult to assess the extent to which these two studies and their findings can be compared, as different cognitive tests were applied. Another interesting aspect of the present study is that impairments in ZNF804A-associated cognitive functions (word reading, word spelling, non-word reading, rapid naming: letters and digits, and phoneme deletion) constitute the characteristic pattern of dyslexia. ${ }^{9}$ Therefore, additional studies of larger samples are required to determine the precise role of ZNF804A in dyslexia per se.

In summary, the same rs1344706 allele in ZNF804A was associated with a better performance in language-related cognitive functions in the present cohort, and was associated with schizophrenia in previous independent studies. If our findings are true, it remains unknown whether these two phenotypes are mediated by the same or different neurobiological pathways, and whether previously reported ZNF804A-imaging findings in healthy individuals ${ }^{10}$ reflect schizophrenia- and/or language-related cognitive processes.

The present findings underline the phenotypic complexity that might be associated with ZNF804A, and illustrate the benefit of testing neurocognitive phenotypes in studies of dyslexia.

\section{Conflict of interest}

The authors declare no conflict of interest.
Acknowledgements. This work was funded by the EU FP6 Program NeuroDys, the Austrian Science Fund (Project 18351-B02) and the Swiss National Science Foundation (Project 32-108130).

1. O'Donovan MC, Craddock N, Norton N, Williams H, Peirce T, Moskvina V et al. Identification of loci associated with schizophrenia by genome-wide association and followup. Nat Genet 2008; 40: 1053-1055

2. Stefansson H, Ophoff RA, Steinberg S, Andreassen OA, Cichon S, Rujescu D et al. Common variants conferring risk of schizophrenia. Nature 2009; 460: 744-747.

3. Gejman PV, Sanders AR, Kendler KS. Genetics of schizophrenia: new findings and challenges. Annu Rev Genomics Hum Genet 2011; 12: 121-144.

4. Leonard CM, Kuldau JM, Maron L, Ricciuti N, Mahoney B, Bengtson M et al. Identical neural risk factors predict cognitive deficit in dyslexia and schizophrenia. Neuropsychology 2008; 22: 147-158.

5. Bersani G, Maneschi ML, Tarolla E, Pancheri P. Dyslexia as a possible aspect of neurocognitive impairment in schizophrenia. Schizophr Res 2006; 82: 265-266.

6. Purcell S, Neale B, Todd-Brown K, Thomas L, Ferreira MA, Bender D et al. PLINK: a tool set for whole-genome association and population-based linkage analyses. Am J Hum Genet 2007; 81: 559-575.

7. Cocchella A, Malacarne M, Forzano F, Marciano C, Pierluigi M, Perroni $L$ et al. The refinement of the critical region for the $2 q 31.2 q 32.3$ deletion syndrome indicates candidate genes for mental retardation and speech impairment. Am J Med Genet B Neuropsychiatr Genet 2010; 153B: 1342-1346.

8. Walters JT, Corvin A, Owen MJ, Williams H, Dragovic M, Quinn EM et al. Psychosis susceptibility gene ZNF804A and cognitive performance in schizophrenia. Arch Gen Psychiatry 2010; 67: 692-700.

9. Vellutino FR, Fletcher JM, Snowling MJ, Scanlon DM. Specific reading disability (dyslexia): what have we learned in the past four decades? J Child Psychol Psychiatry 2004; 45: 2-40.

10. Donohoe G, Morris DW, Corvin A. The psychosis susceptibility gene ZNF804A: associations, functions, and phenotypes. Schizophr Bull 2010; 36: 904-909.

Translational Psychiatry is an open-access journal published by Nature Publishing Group. This work is licensed under the Creative Commons Attribution-Noncommercial-No Derivative Works 3.0 Unported License. To view a copy of this license, visit http://creativecommons.org/licenses/by-nc-nd/3.0/

Supplementary Information accompanies the paper on the Translational Psychiatry website (http://www.nature.com/tp) 